\title{
OPTOELECTRONIC PROPERTIES OF TERNARY TETRAHEDRAL SEMICONDUCTORS
}

\author{
Dajesh C. Gupta ${ }^{a}$, IDjay S. Verma ${ }^{b}, *$, Khushvant Singh $^{\mathrm{a}}$ \\ ${ }^{a}$ Department of Physics, B. S. A. College, Mathura 281004 (India) \\ ${ }^{b}$ Department of Natural and Applied Sciences, School of Technology, Glocal University, Saharanpur 247121 (India) \\ *Corresponding Author: ajay_phy@rediffmail.com \\ Received December 5, 2020; revised February 16, 2021; accepted February 17, 2021
}

The dielectric interpretation of crystal ionicity evolved by Phillips and Van Vechten (P.V.V) has been utilized to evaluate various ground state properties for broad range of semiconductors and insulators. Although, the relevance of P.V.V dielectric theory has been restricted to only simple $\mathrm{A}^{\mathrm{N}} \mathrm{B}^{8-\mathrm{N}}$ structured compounds, which have a particular bond. Levine has broadened P.V.V. theory of ionicity to multiple bond and complex crystals and evaluated many bond parameters for ternary tetrahedral semiconductors. Some other researchers have extended Levine's work with a concept of ionic charge product and nearest neighbour distance to binary and ternary tetrahedral crystals to evaluate the ground state properties. In this paper, a new hypothesis of average atomic number of the elements in a compound has been used to understand the some electronic and optical properties such as ionic gap ( $\mathrm{E}_{\mathrm{c}}$ ), average energy gap $\left(E_{g}\right)$, crystal ionicity $\left(f_{i}\right)$, electronic susceptibility $(\chi)$, and dielectric constant $(\epsilon)$ of ternary tetrahedral $\left(\mathrm{A}^{\mathrm{II}} \mathrm{B}^{\mathrm{IV}} \mathrm{C}_{2}^{\mathrm{V}}\right.$ and $\left.\mathrm{A}^{\mathrm{I}} \mathrm{B}^{\mathrm{III}} \mathrm{C}_{2}^{\mathrm{VI}}\right)$ semiconductors. A reasonably acceptable agreement has been noticed between our evaluated values and other researchers reported values.

KEYWORDS: crystal ionicity, average atomic number, chalcopyrites

Optical and electronic properties of a material are the important parameters for device fabrication in almost all the fields of present-time electronics. The ternary tetrahedral $\left(\mathrm{A}^{\mathrm{II}} \mathrm{B}^{\mathrm{IV}} \mathrm{C}_{2}^{\mathrm{V}}\right.$ and $\left.\mathrm{A}^{\mathrm{I}} \mathrm{B}^{\mathrm{III}} \mathrm{C}_{2}^{\mathrm{VI}}\right)$ structured compounds have brought significant curiosity of researchers' fraternity due to their exciting semiconducting, optical, electrical, mechanical and structural properties. These properties are of underlying significance for the conduct of charge carriers, dopants, impurities and defects in insulators and semiconductors. In contrast to binary analogues these semiconductors have high energy direct band gaps and lower melting points with tetragonal chalcopyrite structure. Structurally chalcopyrite semiconductors are extracted from that of binary sphalerite structure $\left(\mathrm{A}^{\mathrm{II}} \mathrm{B}^{\mathrm{VI}}\right.$ and $\left.\mathrm{A}^{\mathrm{III}} \mathrm{B}^{\mathrm{V}}\right)$ with a slight distortion in the chemical composition. Therefore, similar to their binary analogues ternary tetrahedral semiconductors have a high nonlinear susceptibility. Although due to presence of two different types of bonds in ternary tetrahedral semiconductors they show anisotropicity, which increases high bifringence. High non-linear susceptibility appeared with high bifringence in ternary tetrahedral semiconductors make them very helpful for efficient second harmonic generation and phase matching. Except this, the other major technological applications of these compounds are in photo-voltaic detectors, infrared oscillators, modulators, filters, solar cells, light emitting diodes and lasers etc [1-13].

Theory of crystal ionicity is essential in analyzing the problems in the area of cohesive energy, heat formation, elastic constants, bulk modulus and crystal structure [14]. Van Vechten [15, 16], Phillips [16, 17], Levine [14] and many other workers $[18,19]$ have brought to light various theories and evaluated crystal ionicity for the elementary compounds. Phillips and Van Vechten have computed the heteropolar and homopolar parts to the chemical bond in binary $\mathrm{A}^{\mathrm{N}} \mathrm{B}^{8-\mathrm{N}}$ crystals. Penn [18] suggested one electron model to separate the average energy gap into heteropolar and homopolar parts. Here, homopolar energy gap $\left(E_{h}\right)$ is used as the function of bond length. Levine [14] has broadened Phillips and Van Vechten (P.V.V) theory of ionicity for ternary tetrahedral semiconductors including the effect of ${ }^{\prime} \mathrm{d}^{\text {' }}$ core electrons. Validation of Levine's theory has been given by Singh and Gupta [19]. According to Levine's modified theory of crystal ionicity heteropolar energy gap $\left(\mathrm{E}_{\mathrm{c}}\right)$ depends on bond length and ionic charges involved in bond formation. Kumar et al. [20-22] have evaluated heteropolar and homopolar energy gaps with reference to plasmon energy and it depends directly on the ionic charge of the compound. Ionic charge depends on atomic number of an element in a compound.

Due to difficulties associated with the experimental procedure and its high cost, also intricacies in obtaining correct values of physical properties, researchers have found theoretical methods more appropriate in calculating the physical properties of solids. However due to the lengthy procedure, as well complicated computational methods needed a series of approximation; those methods have always been complex and developed for only certain semiconductors [23]. Hence, we thought it would be of great interest to suggest an another probable interpretation of the ionic gap $\left(E_{c}\right)$, average energy gap $\left(E_{g}\right)$, crystal ionicity $\left(f_{i}\right)$, dielectric constant $(\epsilon)$ and electronic susceptibility $(\chi)$ for ternary tetrahedral $\left(\mathrm{A}^{\mathrm{II}} \mathrm{B}^{\mathrm{IV}} \mathrm{C}_{2}^{\mathrm{V}}\right.$ and $\left.\mathrm{A}^{\mathrm{I}} \mathrm{B}^{\mathrm{III}} \mathrm{C}_{2}^{\mathrm{VI}}\right)$ semiconductors.

Several other researchers have made an effort to compute optical and electronic properties of ternary tetrahedral semiconductors with the help of ionic charge and valance electrons. Besides it considering the different screening factors present in compound crystallography, we have used a new concept of average atomic number of interacting elements in a compound and found excellent outcomes comparable to previous workers for the ionic gap $\left(\mathrm{E}_{\mathrm{c}}\right)$, average energy gap $\left(E_{g}\right)$, crystal ionicity $\left(\mathrm{f}_{\mathrm{i}}\right)$, dielectric constant $(\epsilon)$ and electronic susceptibility $(\chi)$ in ternary tetrahedral C R. C. Gupta, A. S. Verma, K. Singh, 2021 
$\left(\mathrm{A}^{\mathrm{II}} \mathrm{B}^{\mathrm{IV}} \mathrm{C}_{2}^{\mathrm{V}}\right.$ and $\mathrm{A}^{\mathrm{I}} \mathrm{B}^{\mathrm{III}} \mathrm{C}_{2}^{\mathrm{VI}}$ ) semiconductors. In the proposed relations only a few variables such as atomic number and ionic charge on cation and anion are prerequisite; to calculate many electronic and optical properties of these compounds the method emerge relevant to other materials, too.

\section{THEORY, RESULTS AND DISCUSSION}

Covalency and ionicity of the materials have been studied for long as a result researchers have got clear concept of those. The covalent concept involves contribution of electrons between atoms in place of charge transfer, while the ionic concept involves transfer of electron in actual from one atom to another which promotes interaction of two closed shell ions mainly due to short range repulsion and Coulomb force. Pauling [24] has pioneered the concept of crystal ionicity derived from thermo-chemical effect. According to one electron model of Penn [18], the average energy gap $\left(\mathrm{E}_{\mathrm{g}}=\mathrm{E}_{\mathrm{p}}\right)$ may be given as,

$$
\mathrm{E}_{\mathrm{p}}=\hbar \omega_{\mathrm{p}} \mathrm{S}_{\mathrm{o}} /\left(\epsilon_{\infty}-1\right)^{1 / 2}
$$

where $\hbar \omega_{\mathrm{p}}$ is valance electron plasmon energy, $\epsilon_{\infty}$ is the optical dielectric constant and $\mathrm{S}_{\mathrm{o}}=1$.

Plasmon energy of valance electron oscillations both in metal and compound is given as [25],

$$
\hbar \omega_{\mathrm{p}}=28.8 \sqrt{N D / M}
$$

where $\mathrm{N}$ is the number of valence electrons participating in plasma oscillations, $\mathrm{D}$ and $\mathrm{M}$ are density and molecular weight, respectively. Relation (2) is applicable for valance electrons in semiconductors and insulators up to first approximation. Philipp and Ehrenreich [26], and Raether [27] have found that the plasmon energy for semiconductors and insulators is represented by,

$$
\hbar \omega_{\mathrm{pd}}=\hbar \omega_{\mathrm{p}} / \sqrt{1-\delta \varepsilon_{\mathrm{o}}}
$$

where $\delta \varepsilon_{0}$ is a very small correction to valance electron plasmon energy $\hbar \omega_{\mathrm{p}}$ may be overlooked to a first approximation. Philipp and Ehrenreich [26] have expressed that the evaluated values of $\hbar \omega_{\mathrm{p}}$ and $\hbar \omega_{\mathrm{pd}}$ are in good agreement with their observed values of plasmon energy in dielectrics. Kittel [28] has also been shown that plasmon energy in dielectrics is actually the same as in metals.

It is stated in modified theory of dielectric of solids $[15-17,29 ; 19,30]$ for tetrahedral coordinated compounds, average energy gap $\left(\mathrm{E}_{\mathrm{g}}\right)$, relating bonding and anti binding $\left(\mathrm{sp}^{3}\right)$ hybridized orbital can be decomposed into two parts because of the symmetric and anti symmetric contribution to the potential in a unit cell. These two parts are covalent or homopolar gap $\left(\mathrm{E}_{\mathrm{h}}\right)$ and ionic or heteropolar gap $\left(\mathrm{E}_{\mathrm{c}}\right)$, which can be expressed in the following relation:

$$
E_{g}^{2}=E_{c}^{2}+E_{h}^{2}
$$

The ionic or heteroploar gap $\left(\mathrm{E}_{\mathrm{c}}\right)$ is inversely related to the bond length ' $\mathrm{d}$ ' as:

$$
\mathrm{E}_{\mathrm{c}}=\mathrm{K}_{1} \mathrm{~d}^{-1} e^{-K_{s} r_{o}}
$$

where $\mathrm{K}_{1}$ is an arbitrary constant and depends on the difference between valence states of an atom and is given by $\mathrm{K}_{1}=\mathrm{bc}^{2} \Delta z$, where prescreening factor $\mathrm{b}$ depends on the coordination number [14] around the cation i.e. $\mathrm{b}=0.089 N_{C}^{2}$, here $\mathrm{N}_{\mathrm{c}}$ is average coordination number and c-electronic charge, $\Delta z=4$ for IIB chalcogenides. The values of $\mathrm{N}_{\mathrm{c}}=4$, $\mathrm{b}=1.424$ and hence $\mathrm{K}_{1}=262.784$ are for zinc blende type structure. Thomas-Fermi screening parameter $\mathrm{K}_{\mathrm{s}}$ has been ascertained taking into account eight electrons per molecule (two for $\mathrm{Zn}, \mathrm{Cd} \& \mathrm{Hg}$ and six for $\mathrm{O}, \mathrm{S}, \mathrm{Se} \& \mathrm{Te}$ ) and $\mathrm{r}_{\mathrm{o}}=$ $\mathrm{d} / 2$.

The covalent or homopolar gap $\left(E_{h}\right)$ is related to the bond length' $d$ ' as [16]:

$$
\mathrm{E}_{\mathrm{h}}=\mathrm{Ad}^{-K_{2}}
$$

Here $\mathrm{A}$ and $\mathrm{K}_{2}$ are constants, which remain unaffected in different crystal structures with values $\mathrm{A}=40.468 \mathrm{eV} / \AA^{2.5}$ and $K_{2}=2.5$. These values are $A=39.74$ and $K_{2}=2.48$ as reported by PVV theory. Therefore, the values of $E_{c}$ and $E_{h}$ depend on number of bonds emerge from cations and bond length.

The crystal ionicity $f_{i}$ of the chemical bond is expressed as the fraction of ionic character as follows.

$$
\mathrm{f}_{\mathrm{i}}=E_{c}^{2} / E_{g}^{2}
$$

V. Kumar [20-22] has expressed those electronic properties of ternary tetrahedral $\left(\mathrm{A}^{\mathrm{II}} \mathrm{B}^{\mathrm{IV}} \mathrm{C}_{2}^{\mathrm{V}}\right.$ and $\left.\mathrm{A}^{\mathrm{I}} \mathrm{B}^{\mathrm{III}} \mathrm{C}_{2}^{\mathrm{VI}}\right)$ crystals in terms of plasmon energy $\hbar \omega_{\mathrm{p}}(\mathrm{eV})$ as follows:

For the $\mathrm{A}-\mathrm{C}$ bond in $\mathrm{A}^{\mathrm{II}} \mathrm{B}^{\mathrm{IV}} \mathrm{C}_{2}^{\mathrm{V}}$ :

$$
\begin{gathered}
\mathrm{E}_{\mathrm{h}, \mathrm{AC}}(\mathrm{eV})=0.05118\left(\hbar \omega_{\mathrm{p}, \mathrm{AC}}\right)^{v}, \\
\mathrm{E}_{\mathrm{c}, \mathrm{AC}}(\mathrm{eV})=5.904 \mathrm{~b}_{\mathrm{AC}}\left(\hbar \omega_{\mathrm{p}, \mathrm{AC}}\right)^{\mu} \times \exp \left[-5.971\left(\hbar \omega_{\mathrm{p}, \mathrm{AC}}\right)^{-\mu / 2}\right]
\end{gathered}
$$


For the $\mathrm{B}-\mathrm{C}$ bond in $\mathrm{A}^{\mathrm{II}} \mathrm{B}^{\mathrm{IV}} \mathrm{C}_{2}^{\mathrm{V}}$ :

$$
\begin{gathered}
\mathrm{E}_{\mathrm{h}, \mathrm{BC}}(\mathrm{eV})=0.04158\left(\hbar \omega_{\mathrm{p}, \mathrm{BC}}\right)^{v} \\
\mathrm{E}_{\mathrm{c}, \mathrm{BC}}(\mathrm{eV})=1.81 \mathrm{~b}_{\mathrm{BC}}\left(\hbar \omega_{\mathrm{p}, \mathrm{BC}}\right)^{\mu} \times \exp \left[-6.4930\left(\hbar \omega_{\mathrm{p}, \mathrm{BC}}\right)^{-\mu / 2}\right]
\end{gathered}
$$

For the $\mathrm{A}-\mathrm{C}$ bond in $\mathrm{A}^{\mathrm{I}} \mathrm{B}^{\mathrm{III}} \mathrm{C}_{2}^{\mathrm{VI}}$ :

$$
\begin{gathered}
\mathrm{E}_{\mathrm{h}, \mathrm{AC}}(\mathrm{eV})=0.0246\left(\hbar_{\mathrm{p}, \mathrm{AC}}\right)^{v}, \\
\mathrm{E}_{\mathrm{c}, \mathrm{AC}}(\mathrm{eV})=7.3208 \mathrm{~b}_{\mathrm{AC}}\left(\hbar \omega_{\mathrm{p}, \mathrm{AC}}\right)^{2 / 3} \times \exp \left[-8.026\left(\hbar \omega_{\mathrm{p}, \mathrm{AC}}^{\prime}\right)^{-1 / 3}\left(\left(\hbar \omega_{\mathrm{p}, \mathrm{AC}}\right)^{-2 / 3}\right]\right.
\end{gathered}
$$

For the $\mathrm{B}-\mathrm{C}$ bond in $\mathrm{A}^{\mathrm{I}} \mathrm{B}^{\mathrm{III}} \mathrm{C}_{2}^{\mathrm{VI}}$ :

$$
\begin{gathered}
\mathrm{E}_{\mathrm{h}, \mathrm{BC}}(\mathrm{eV})=0.0416\left(\hbar \omega_{\mathrm{p}, \mathrm{BC}}\right)^{v}, \\
\mathrm{E}_{\mathrm{c}, \mathrm{BC}}(\mathrm{eV})=5.53 \mathrm{~b}_{\mathrm{BC}}\left(\hbar \omega_{\mathrm{p}, \mathrm{BC}}\right)^{2 / 3} \times \exp \left[-6.5058\left(\hbar \omega_{\mathrm{p}, \mathrm{BC}}\right)^{-2 / 3}\right]
\end{gathered}
$$

where $v$ is a constant. $E_{c}$ and $E_{h}$ are the ionic and homopolar gaps, respectively. The authors of above theory have used constants $\mu, v$ and prescreening factor $\mathrm{b}$ to evaluate the parameters mentioned therein.

Jayaraman et al. [31], Srideshmukh et al. [32], and Krishnan et al. [33] established that significantly reduced ionic charges must be used to obtain better agreement with experimental values of these parameters. Verma et al. [34] have been applied this concept for obtaining better agreement with theoretical data for ionic gap $\left(\mathrm{E}_{\mathrm{c}}\right)$, average energy gap $\left(\mathrm{E}_{\mathrm{g}}\right)$, crystal ionicity $\left(\mathrm{f}_{\mathrm{i}}\right)$ dielectric constant $(\epsilon)$ and susceptibility $(\chi)$ of ternary tetrahedral $\left(\mathrm{A}^{\mathrm{II}} \mathrm{B}^{\mathrm{IV}} \mathrm{C}_{2}^{\mathrm{V}}\right.$ and $\left.\mathrm{A}^{\mathrm{I}} \mathrm{B}^{\mathrm{III}} \mathrm{C}_{2}^{\mathrm{VI}}\right)$ semiconductors, which can be expressed in terms of product of ionic charges and bond length as follows:

For A-C bond in ternary tetrahedral $\left(\mathrm{A}^{\mathrm{II}} \mathrm{B}^{\mathrm{IV}} \mathrm{C}_{2}^{\mathrm{V}}\right.$ and $\left.\mathrm{A}^{\mathrm{I}} \mathrm{B}^{\mathrm{III}} \mathrm{C}_{2}^{\mathrm{VI}}\right)$ semiconductors:

$$
\begin{gathered}
\mathrm{E}_{\mathrm{c}, \mathrm{AC}}(\mathrm{eV})=90 d^{2} /\left(Z_{1} Z_{2}\right)^{0.7}, \\
\mathrm{E}_{\mathrm{g}, \mathrm{AC}}(\mathrm{eV})=96 d^{2.12} /\left(Z_{1} Z_{2}\right)^{0.5}
\end{gathered}
$$

For B-C bond in ternary tetrahedral $\left(\mathrm{A}^{\mathrm{II}} \mathrm{B}^{\mathrm{IV}} \mathrm{C}_{2}^{\mathrm{V}}\right.$ and $\left.\mathrm{A}^{\mathrm{I}} \mathrm{B}^{\mathrm{III}} \mathrm{C}_{2}^{\mathrm{VI}}\right)$ semiconductors:

$$
\begin{gathered}
\mathrm{E}_{\mathrm{c}, \mathrm{BC}}(\mathrm{eV})=275 d^{2} /\left(Z_{1} Z_{2}\right)^{1.25}, \\
\mathrm{E}_{\mathrm{g}, \mathrm{BC}}(\mathrm{eV})=106 d^{2.12} /\left(Z_{1} Z_{2}\right)^{0.5}
\end{gathered}
$$

where, $Z_{1}$ and $Z_{2}$ are the ionic charges and $d$ is the bond length in $\AA$. On solving equations (7) and (16-19), crystal ionicity $f_{i}$ can be expressed as follows:

For A-C bond in ternary tetrahedral $\left(\mathrm{A}^{\mathrm{II}} \mathrm{B}^{\mathrm{IV}} \mathrm{C}_{2}^{\mathrm{V}}\right.$ and $\left.\mathrm{A}^{\mathrm{I}} \mathrm{B}^{\mathrm{III}} \mathrm{C}_{2}^{\mathrm{VI}}\right)$ semiconductors:

$$
\mathrm{f}_{\mathrm{i}}=0.87891 d^{0.24} /\left(Z_{1} Z_{2}\right)^{0.4}
$$

For B-C bond in ternary tetrahedral $\left(\mathrm{A}^{\mathrm{II}} \mathrm{B}^{\mathrm{IV}} \mathrm{C}_{2}^{\mathrm{V}}\right.$ and $\left.\mathrm{A}^{\mathrm{I}} \mathrm{B}^{\mathrm{III}} \mathrm{C}_{2}^{\mathrm{VI}}\right)$ semiconductors:

$$
\mathrm{f}_{\mathrm{i}}=6.731 d^{0.24} /\left(Z_{1} Z_{2}\right)^{1.5}
$$

V. Kumar [35] has given the relation for electronic susceptibility $\chi$ as follows:

$$
\chi_{X Y}=\left(\hbar \omega_{p, X Y}\right)^{2} /\left(E_{g, X Y}\right)^{2},
$$

and dielectric constant

$$
\epsilon_{X Y}=1+\chi_{X Y} .
$$

As, the plasmon energy $\left(\hbar \omega_{\mathrm{p}}\right.$ ) and ionic charge depend on the number of valance electrons, which depend on atomic number in an atom. But in a compound this may depend on the atomic numbers of the constituent atoms. Hence, we have proposed the idea of average atomic number of the atoms in a compound to study the ground state properties. As, this parameter of average atomic number includes all the distribution laws of electrons in an atom and various screening factors. So, taking into account the above empirical relations and applying the concept of product of ionic charges given by Verma at el. [34] and our hypothesis of average atomic number of elements in the ternary tetrahedral semiconductors, the crystal ionicity $\left(\mathrm{f}_{\mathrm{i}}\right)$ of ternary tetrahedral semiconductors manifests a nearly linear dependence when traced against average atomic number. 
However crystal ionicity falls on two closely parallel straight lines according to the product of ionic charge of the compounds and this is presented in the Fig. 1 and 2. Applying this concept to reach better agreement with theoretical data, we have introduced following empirical relations for electronic and optical properties such as ionic gap $\left(E_{c}\right)$, average energy gap $\left(E_{g}\right)$, crystal ionicity $\left(f_{i}\right)$, electronic susceptibility $(\chi)$ and dielectric constant $(\epsilon)$ and further evaluated their values. The evaluated values are in excellent agreement with the values reported by Levine [14], V. Kumar $[21,35,36]$. Our endeavour of determining those has not been required experimental values of the dielectric constant although the previous models needed this value in their computations except Verma et al. [34]. These values are presented in the Table 1-5.

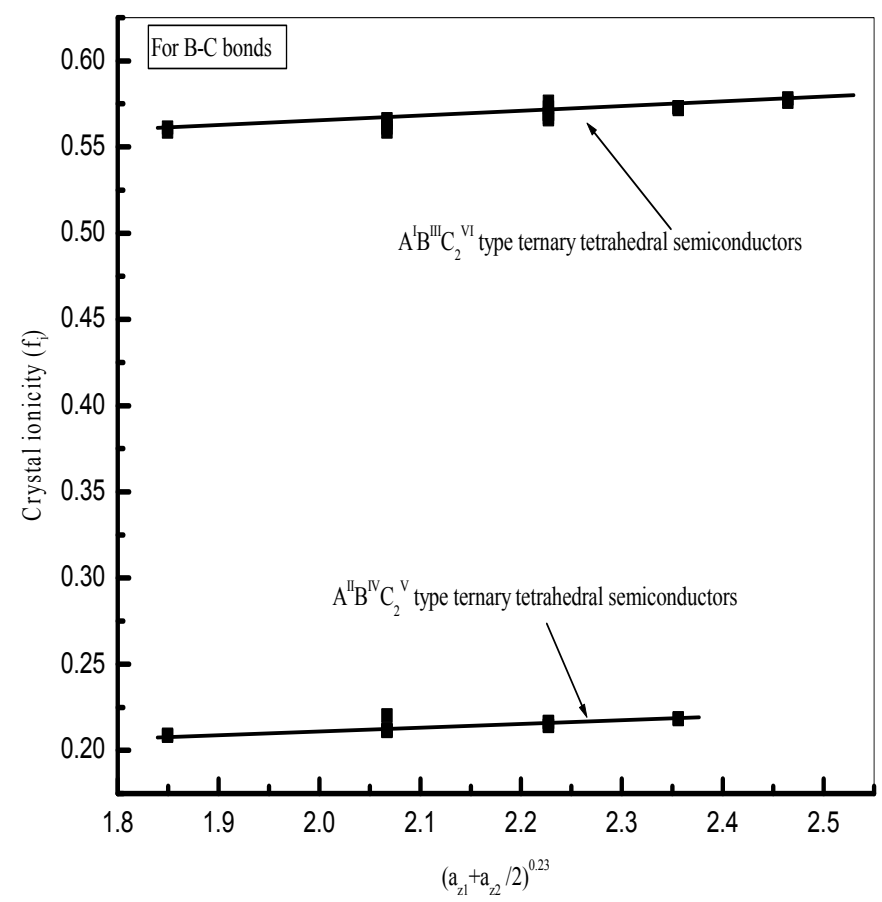

Figure 1. Plot of crystal ionicity versus average atomic number for $\mathrm{A}-\mathrm{C}$ bonds in ternary tetrahedral $\left(\mathrm{A}^{\mathrm{II}} \mathrm{B}^{\mathrm{IV}} \mathrm{C}_{2}^{\mathrm{V}}\right.$ and $\left.\mathrm{A}^{\mathrm{I}} \mathrm{B}^{\mathrm{III}} \mathrm{C}_{2}^{\mathrm{VI}}\right)$ semiconductors. Here, crystal ionicity of $\mathrm{A}^{\mathrm{II}} \mathrm{B}^{\mathrm{IV}} \mathrm{C}_{2}^{\mathrm{V}}$ lies on a line closely parallel to the line of $\mathrm{A}^{\mathrm{I}} \mathrm{B}^{\mathrm{III}} \mathrm{C}_{2}^{\mathrm{VI}}$. In this figure values of $\left(\mathrm{f}_{\mathrm{i}}\right.$ ) taken from V. Kumar $[35,36]$.

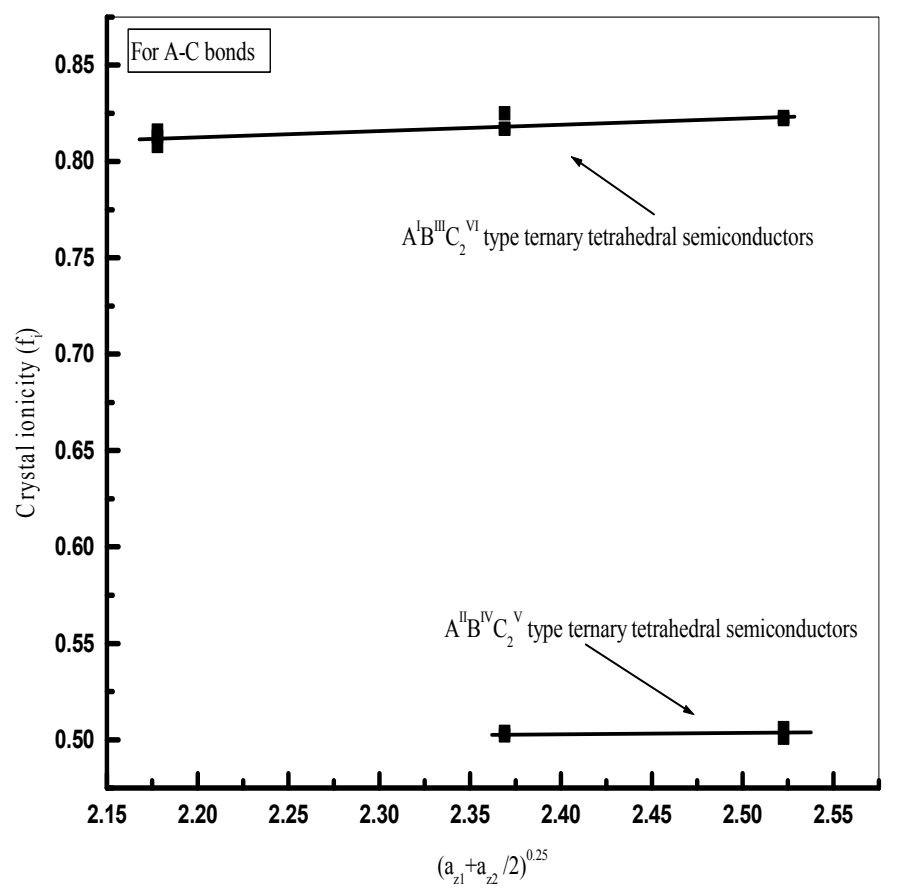

Figure 2. Plot of crystal ionicity versus average atomic number for $\mathrm{B}-\mathrm{C}$ bonds in ternary tetrahedral $\left(\mathrm{A}^{\mathrm{II}} \mathrm{B}^{\mathrm{IV}} \mathrm{C}_{2}^{\mathrm{V}}\right.$ and $\left.\mathrm{A}^{\mathrm{I}} \mathrm{B}^{\mathrm{III}} \mathrm{C}_{2}^{\mathrm{VI}}\right)$ semiconductors. Here, crystal ionicity of $\mathrm{A}^{\mathrm{II}} \mathrm{B}^{\mathrm{IV}} \mathrm{C}_{2}^{\mathrm{V}}$ lies on a line closely parallel to the line of $\mathrm{A}^{\mathrm{I}} \mathrm{B}^{\mathrm{III}} \mathrm{C}_{2}^{\mathrm{VI}}$. In this figure values of $\left(\mathrm{f}_{\mathrm{i}}\right.$ ) taken from V. Kumar $[35,36]$.

For A-C bond in ternary tetrahedral $\left(\mathrm{A}^{\mathrm{II}} \mathrm{B}^{\mathrm{IV}} \mathrm{C}_{2}^{\mathrm{V}}\right.$ and $\left.\mathrm{A}^{\mathrm{I}} \mathrm{B}^{\mathrm{III}} \mathrm{C}_{2}^{\mathrm{VI}}\right)$ semiconductors: 


$$
\begin{gathered}
\mathrm{E}_{\mathrm{c}, \mathrm{AC}}(\mathrm{eV})=88 /\left(Z_{1} Z_{2}\right)^{0.8}\left(\frac{a_{Z 1}+a_{Z 2}}{2}\right)^{0.5} \\
\mathrm{E}_{\mathrm{g}, \mathrm{AC}}(\mathrm{eV})=95 /\left(Z_{1} Z_{2}\right)^{0.8}\left(\frac{a_{Z 1}+a_{Z 2}}{2}\right)^{0.5} \\
\mathrm{f}_{\mathrm{i}, \mathrm{AC}}=0.4\left(\frac{a_{\mathrm{z} 1}+a_{\mathrm{Z} 2}}{2}\right)^{0.25} /\left(Z_{1} Z_{2}\right)^{0.3}
\end{gathered}
$$

For $\mathrm{B}-\mathrm{C}$ bond in ternary tetrahedral $\left(\mathrm{A}^{\mathrm{II}} \mathrm{B}^{\mathrm{IV}} \mathrm{C}_{2}^{\mathrm{V}}\right.$ and $\left.\mathrm{A}^{\mathrm{I}} \mathrm{B}^{\mathrm{III}} \mathrm{C}_{2}^{\mathrm{VI}}\right)$ semiconductors:

$$
\begin{gathered}
\mathrm{E}_{\mathrm{c}, \mathrm{BC}}(\mathrm{eV})=90 /\left(Z_{1} Z_{2}\right)^{0.8}\left(\frac{a_{Z 1}+a_{Z 2}}{2}\right)^{0.49}, \\
\mathrm{E}_{\mathrm{g}, \mathrm{BC}}(\mathrm{eV})=122 /\left(Z_{1} Z_{2}\right)^{0.8}\left(\frac{a_{Z 1}+a_{Z 2}}{2}\right)^{0.43} \\
\mathrm{f}_{\mathrm{i}, \mathrm{BC}}=4\left(\frac{a_{z 1}+a_{z 2}}{2}\right)^{0.23} /\left(Z_{1} Z_{2}\right)^{1.5}
\end{gathered}
$$

\begin{tabular}{|c|c|c|c|c|c|c|c|c|c|c|}
\hline Solids & $a_{\mathrm{z} 1}$ & $a_{\mathrm{z} 2}$ & $\mathrm{E}_{\mathrm{c}}[14]$ & $\mathrm{E}_{\mathrm{c}}[35,36]$ & $E_{c}[34]$ & $E_{c}[$ This work] & $\mathrm{Eg}_{\mathrm{g}}[14]$ & $E_{g}[35,36]$ & $\mathrm{Eg}_{\mathrm{g}}[34]$ & $\mathrm{E}_{\mathrm{g}}$ [This work] \\
\hline $\mathrm{CuAlS}_{2}$ & 29 & 16 & & 10.575 & 10.565 & 10.655 & & 11.728 & 11.719 & 12.941 \\
\hline $\mathrm{CuAlSe}_{2}$ & 29 & 34 & & 9.435 & 9.46 & 9.005 & & 10.436 & 10.425 & 10.938 \\
\hline $\mathrm{CuAlTe}_{2}$ & 29 & 52 & & 8.035 & 8.196 & 7.942 & & 8.859 & 8.954 & 9.646 \\
\hline $\mathrm{CuGaS}_{2}$ & 29 & 16 & 9.95 & 9.673 & 9.699 & 10.655 & 10.964 & 10.705 & 10.704 & 12.941 \\
\hline $\mathrm{CuGaSe}_{2}$ & 29 & 34 & 7.87 & 8.807 & 8.936 & 9.005 & 8.888 & 9.692 & 9.813 & 10.938 \\
\hline $\mathrm{CuGaTe}_{2}$ & 29 & 52 & & 7.945 & 8.133 & 7.942 & & 8.758 & 8.881 & 9.646 \\
\hline $\mathrm{CuInS}_{2}$ & 29 & 16 & 8.52 & 8.523 & 8.936 & 10.655 & 9.617 & 9.478 & 9.813 & 12.941 \\
\hline $\mathrm{CuInSe}_{2}$ & 29 & 34 & & 9.333 & 9.382 & 9.005 & & 10.321 & 10.334 & 10.938 \\
\hline $\mathrm{CuInTe}_{2}$ & 29 & 52 & & 6.635 & 7.83 & 7.942 & & 7.516 & 8.531 & 9.646 \\
\hline $\mathrm{AgAlS}_{2}$ & 47 & 16 & & 9.32 & 8.587 & 9.005 & & 10.117 & 9.408 & 10.938 \\
\hline $\mathrm{AgAlSe}_{2}$ & 47 & 34 & & 8.393 & 7.83 & 7.942 & & 9.096 & 8.531 & 9.646 \\
\hline $\operatorname{AgAlTe}_{2}$ & 47 & 52 & & 7.51 & 7.117 & 7.184 & & 8.128 & 7.71 & 8.725 \\
\hline $\mathrm{AgGaS}_{2}$ & 47 & 16 & 9.87 & 9.103 & 8.388 & 9.005 & 10.6 & 9.877 & 9.177 & 10.938 \\
\hline $\mathrm{AgGaSe}_{2}$ & 47 & 34 & 8.56 & 8.405 & 7.83 & 7.942 & 9.275 & 9.11 & 8.531 & 9.646 \\
\hline $\mathrm{AgGaTe}_{2}$ & 47 & 52 & & 7.649 & 7.22 & 7.184 & & 8.281 & 7.829 & 8.725 \\
\hline $\mathrm{AgInS}_{2}$ & 47 & 16 & & 9.608 & 8.794 & 9.005 & & 10.434 & 9.648 & 10.938 \\
\hline $\mathrm{AgInSe}_{2}$ & 47 & 34 & 8.15 & 8.662 & 8.01 & 7.942 & 8.862 & 9.392 & 8.739 & 9.646 \\
\hline $\operatorname{AgInTe}_{2}$ & 47 & 52 & & 7.554 & 7.117 & 7.184 & & 8.176 & 7.71 & 8.725 \\
\hline $\mathrm{ZnSiP}_{2}$ & 30 & 15 & 4.1 & 4.584 & 4.552 & 4.425 & 6.119 & 6.53 & 6.263 & 6.477 \\
\hline $\mathrm{ZnGeP}_{2}$ & 30 & 15 & 4.08 & 4.541 & 4.499 & 4.425 & 6.134 & 6.465 & 6.186 & 6.477 \\
\hline $\mathrm{ZnSnP}_{2}$ & 30 & 15 & 4.07 & 4.414 & 4.399 & 4.425 & 6.038 & 6.273 & 6.04 & 6.477 \\
\hline $\mathrm{ZnSiAs}_{2}$ & 30 & 33 & 3.73 & 4.225 & 4.229 & 3.739 & 5.655 & 5.989 & 5.793 & 5.474 \\
\hline $\mathrm{ZnGeAs} 2$ & 30 & 33 & 3.71 & 4.157 & 4.168 & 3.739 & 5.582 & 5.887 & 5.705 & 5.474 \\
\hline $\mathrm{ZnSnAs}_{2}$ & 30 & 33 & 3.68 & 4.061 & 4.082 & 3.739 & 5.48 & 5.744 & 5.58 & 5.474 \\
\hline $\mathrm{CdSiP}_{2}$ & 48 & 15 & 4.17 & 3.875 & 3.915 & 3.739 & 5.682 & 5.647 & 5.338 & 5.474 \\
\hline $\mathrm{CdGeP}_{2}$ & 48 & 15 & 4.12 & 3.882 & 3.921 & 3.739 & 5.652 & 5.478 & 5.347 & 5.474 \\
\hline $\mathrm{CdSnP}_{2}$ & 48 & 15 & 4.04 & 3.785 & 3.834 & 3.739 & 5.519 & 5.334 & 5.221 & 5.474 \\
\hline $\mathrm{CdSiAs}_{2}$ & 48 & 33 & 3.98 & 3.618 & 3.684 & 3.298 & 5.354 & 5.088 & 5.005 & 4.828 \\
\hline $\mathrm{CdGeAs}_{2}$ & 48 & 33 & 3.94 & 3.612 & 3.679 & 3.298 & 5.317 & 5.08 & 4.997 & 4.828 \\
\hline CdSnAs2 & 48 & 33 & 3.87 & 3.527 & 3.602 & 3.298 & 5.205 & 4.983 & 4.887 & 4.828 \\
\hline
\end{tabular}

while, we have found electronic susceptibility $\left(\chi_{\mathrm{xy}}\right)$ same as given in relation $(22)$ and can be calculated for $\mathrm{AC}$ and $\mathrm{BC}$ bonds by using the values of $E_{g}$ obtained from relations (25) and (28), thereafter dielectric constant $\left(\epsilon_{\mathrm{xy}}\right)$ by the relation (23). Here, $Z_{1}, Z_{2}$ are ionic charges and $a_{z 1}, a_{Z 2}$ are atomic numbers of the elements in the compound.

Table 1. This table presents the values of ionic gaps $\mathrm{E}_{\mathrm{c}}(\mathrm{eV})$ and average energy gaps $\mathrm{E}_{\mathrm{g}}(\mathrm{eV})$ for A-C bond of ternary tetrahedral $\left(\mathrm{A}^{\mathrm{I}} \mathrm{B}^{\mathrm{III}} \mathrm{C}_{2}^{\mathrm{VI}}\right.$ and $\mathrm{A}^{\mathrm{II}} \mathrm{B}^{\mathrm{IV}} \mathrm{C}_{2}^{\mathrm{V}}$ ) semiconductors. 
Table 2. This table presents the values of ionic gaps $\mathrm{E}_{\mathrm{c}}(\mathrm{eV})$ and average energy gaps $\mathrm{E}_{\mathrm{g}}(\mathrm{eV})$ for $\mathrm{B}-\mathrm{C}$ bond of ternary tetrahedral $\left(\mathrm{A}^{\mathrm{I}} \mathrm{B}^{\mathrm{III}} \mathrm{C}_{2}^{\mathrm{VI}}\right.$ and $\left.\mathrm{A}^{\mathrm{II}} \mathrm{B}^{\mathrm{IV}} \mathrm{C}_{2}^{\mathrm{V}}\right)$ semiconductors.

\begin{tabular}{|c|c|c|c|c|c|c|c|c|c|c|}
\hline Solids & $\mathrm{a}_{\mathrm{z} 1}$ & $\mathrm{a}_{\mathrm{z} 2}$ & $\mathrm{E}_{\mathrm{c}}[14]$ & $\mathrm{E}_{\mathrm{c}}[35,36]$ & $\mathrm{E}_{\mathrm{c}}[34]$ & $\mathrm{E}_{\mathrm{c}}$ [This work] & Eg [14] & $E_{g}[35,36]$ & Eg [34] & $\mathrm{E}_{\mathrm{g}}[$ This work $]$ \\
\hline $\mathrm{CuAlS}_{2}$ & 29 & 16 & & 5.746 & 5.584 & 5.790 & & 7.671 & 7.471 & 9.214 \\
\hline $\mathrm{CuAlSe}_{2}$ & 29 & 34 & & 5.165 & 5.084 & 4.570 & & 6.863 & 6.764 & 7.487 \\
\hline $\mathrm{CuAlTe}_{2}$ & 29 & 52 & & 4.479 & 4.469 & 3.899 & & 5.902 & 5.899 & 6.512 \\
\hline $\mathrm{CuGaS}_{2}$ & 29 & 16 & 5.6 & 5.684 & 5.536 & 4.570 & 7.45 & 7.584 & 7.402 & 7.487 \\
\hline $\mathrm{CuGaSe}_{2}$ & 29 & 34 & 5.1 & 5.103 & 5.001 & 3.899 & 6.8 & 6.777 & 6.646 & 6.512 \\
\hline $\mathrm{CuGaTe}_{2}$ & 29 & 52 & & 4.336 & 4.366 & 3.458 & & 5.725 & 5.755 & 5.862 \\
\hline $\mathrm{CuInS}_{2}$ & 29 & 16 & 5.49 & 4.898 & 4.839 & 3.899 & 6.39 & 6.494 & 6.419 & 6.512 \\
\hline $\mathrm{CuInSe}{ }_{2}$ & 29 & 34 & & 4.466 & 4.469 & 3.458 & & 5.902 & 5.899 & 5.862 \\
\hline $\mathrm{CuInTe}_{2}$ & 29 & 52 & & 3.96 & 4.017 & 3.141 & & 5.215 & 5.269 & 5.388 \\
\hline $\mathrm{AgAlS}_{2}$ & 47 & 16 & & 5.939 & 5.734 & 5.790 & & 7.943 & 7.683 & 9.214 \\
\hline $\mathrm{AgAlSe}_{2}$ & 47 & 34 & & 5.381 & 5.258 & 4.570 & & 7.162 & 7.009 & 7.487 \\
\hline $\mathrm{AgAlTe}_{2}$ & 47 & 52 & & 4.43 & 4.434 & 3.899 & & 5.853 & 5.85 & 6.512 \\
\hline $\mathrm{AgGaS}_{2}$ & 47 & 16 & 5.64 & 5.887 & 5.683 & 4.570 & 7.47 & 7.869 & 7.611 & 7.487 \\
\hline $\mathrm{AgGaSe}_{2}$ & 47 & 34 & 5.02 & 5.235 & 5.127 & 3.899 & 6.7 & 6.959 & 6.824 & 6.512 \\
\hline $\mathrm{AgGaTe}_{2}$ & 47 & 52 & & 4.316 & 4.332 & 3.458 & & 5.698 & 5.708 & 5.862 \\
\hline $\mathrm{AgInS}_{2}$ & 47 & 16 & & 4.835 & 4.8 & 3.899 & & 6.408 & 6.364 & 6.512 \\
\hline $\mathrm{AgInSe}_{2}$ & 47 & 34 & 4.71 & 4.378 & 4.4 & 3.458 & 6.7 & 5.783 & 5.802 & 5.862 \\
\hline $\mathrm{AgInTe}_{2}$ & 47 & 52 & & 3.758 & 3.844 & 3.141 & & 4.944 & 5.029 & 5.388 \\
\hline $\mathrm{ZnSiP}_{2}$ & 30 & 15 & 2.45 & 2.72 & 2.424 & 3.325 & 5.84 & 5.95 & 5.463 & 5.292 \\
\hline $\mathrm{ZnGeP}_{2}$ & 30 & 15 & 2.6 & 2.546 & 2.28 & 2.625 & 5.56 & 5.54 & 5.12 & 4.300 \\
\hline $\mathrm{ZnSnP}_{2}$ & 30 & 15 & 2.71 & 2.183 & 1.994 & 2.239 & 4.97 & 4.7 & 4.443 & 3.740 \\
\hline ZnSiAs2 & 30 & 33 & 2.54 & 2.478 & 2.23 & 2.625 & 5.41 & 5.38 & 5.001 & 4.300 \\
\hline $\mathrm{ZnGeAs}_{2}$ & 30 & 33 & 2.12 & 2.347 & 2.125 & 2.239 & 4.97 & 5.07 & 4.753 & 3.740 \\
\hline $\mathrm{ZnSnAs}_{2}$ & 30 & 33 & 1.53 & 2.045 & 1.883 & 1.986 & 4.16 & 4.38 & 4.182 & 3.367 \\
\hline $\mathrm{CdSiP}_{2}$ & 48 & 15 & 2.59 & 2.739 & 2.439 & 3.325 & 5.94 & 6 & 5.5 & 5.292 \\
\hline $\mathrm{CdGeP}_{2}$ & 48 & 15 & 2.68 & 2.538 & 2.278 & 2.625 & 5.59 & 5.52 & 5.116 & 4.300 \\
\hline $\mathrm{CdSnP}_{2}$ & 48 & 15 & 2.71 & 2.181 & 1.992 & 2.239 & 4.96 & 4.69 & 4.439 & 3.740 \\
\hline $\mathrm{CdSiAs}_{2}$ & 48 & 33 & 2.64 & 2.543 & 2.233 & 2.625 & 5.46 & 5.42 & 5.01 & 4.300 \\
\hline $\mathrm{CdGeAs}_{2}$ & 48 & 33 & 2.21 & 2.317 & 2.101 & 2.239 & 4.95 & 5 & 4.695 & 3.740 \\
\hline $\mathrm{CdSnAs}_{2}$ & 48 & 33 & 1.59 & 2.017 & 1.861 & 1.986 & 4.14 & 4.32 & 4.13 & 3.367 \\
\hline
\end{tabular}

Table 3. This table presents the values of crystal ionicity fif for $A-C$ and $B-C$ bonds of ternary tetrahedral $\left(\mathrm{A}^{\mathrm{I}} \mathrm{B}^{\mathrm{III}} \mathrm{C}_{2}^{\mathrm{VI}}\right.$ and $\left.\mathrm{A}^{\mathrm{II}} \mathrm{B}^{\mathrm{IV}} \mathrm{C}_{2}^{\mathrm{V}}\right)$ semiconductors.

\begin{tabular}{|c|c|c|c|c|c|c|c|c|c|c|}
\hline \multirow{2}{*}{ Solids } & $\begin{array}{c}f_{i} \\
{[14]}\end{array}$ & $\begin{array}{c}f_{i} \\
{[35,36]} \\
\end{array}$ & $\begin{array}{c}f_{i} \\
{[37]} \\
\end{array}$ & $\begin{array}{c}f_{i} \\
{[34]}\end{array}$ & $\begin{array}{c}f_{i} \\
\text { [This work] }\end{array}$ & $\begin{array}{c}f_{i} \\
{[14]}\end{array}$ & $\begin{array}{c}f_{i} \\
{[35,36]}\end{array}$ & $\begin{array}{c}f_{i} \\
{[37]}\end{array}$ & $\begin{array}{c}f_{i} \\
{[34]}\end{array}$ & $\begin{array}{c}f_{i} \\
\text { [This work] }\end{array}$ \\
\hline & \multicolumn{5}{|c|}{ A-C Bond } & \multicolumn{5}{|c|}{ B-C Bond } \\
\hline $\mathrm{CuAlS}_{2}$ & \multirow{6}{*}{$\begin{array}{l}0.825 \\
0.784\end{array}$} & 0.813 & 0.78 & 0.813 & 0.708 & \multirow{6}{*}{$\begin{array}{l}0.565 \\
0.563\end{array}$} & 0.561 & 0.62 & 0.559 & 0.503 \\
\hline $\mathrm{CuAlSe}_{2}$ & & 0.817 & 0.79 & 0.824 & 0.770 & & 0.566 & 0.65 & 0.565 & 0.563 \\
\hline $\mathrm{CuAlTe}_{2}$ & & 0.822 & 0.75 & 0.838 & 0.820 & & 0.576 & 0.56 & 0.574 & 0.606 \\
\hline $\mathrm{CuGaS}_{2}$ & & 0.816 & 0.77 & 0.821 & 0.708 & & 0.561 & 0.55 & 0.559 & 0.563 \\
\hline $\mathrm{CuGaSe}_{2}$ & & 0.825 & 0.76 & 0.829 & 0.770 & & 0.567 & 0.55 & 0.566 & 0.606 \\
\hline $\mathrm{CuGaTe}_{2}$ & & 0.823 & 0.81 & 0.839 & 0.820 & & 0.573 & 0.51 & 0.576 & 0.641 \\
\hline $\mathrm{CuInS}_{2}$ & \multirow[t]{6}{*}{0.785} & 0.808 & 0.77 & 0.829 & 0.708 & \multirow[t]{6}{*}{0.636} & 0.569 & 0.6 & 0.569 & 0.606 \\
\hline $\mathrm{CuInSe} 2$ & & 0.817 & 0.77 & 0.824 & 0.770 & & 0.572 & 0.6 & 0.574 & 0.641 \\
\hline $\mathrm{CuInTe} 2$ & & 0.779 & 0.76 & 0.842 & 0.820 & & 0.576 & 0.57 & 0.581 & 0.671 \\
\hline $\mathrm{AgAlS}_{2}$ & & 0.848 & 0.85 & 0.833 & 0.770 & & 0.559 & 0.61 & 0.557 & 0.503 \\
\hline $\mathrm{AgAlSe}_{2}$ & & 0.851 & 0.86 & 0.842 & 0.820 & & 0.564 & 0.63 & 0.563 & 0.563 \\
\hline $\mathrm{AgAlTe}_{2}$ & & 0.853 & 0.84 & 0.852 & 0.862 & & 0.573 & 0.57 & 0.575 & 0.606 \\
\hline
\end{tabular}




\begin{tabular}{|c|c|c|c|c|c|c|c|c|c|c|}
\hline \multirow[t]{2}{*}{ Solids } & $\begin{array}{c}f_{i} \\
{[14]}\end{array}$ & $\begin{array}{c}f_{i} \\
{[35,36]}\end{array}$ & $\begin{array}{c}\mathrm{f}_{\mathrm{i}} \\
{[37]}\end{array}$ & $\begin{array}{c}f_{i} \\
{[34]}\end{array}$ & $\begin{array}{c}f_{i} \\
\text { [This work] }\end{array}$ & $\begin{array}{c}f_{i} \\
{[14]}\end{array}$ & $\begin{array}{c}f_{i} \\
{[35,36]}\end{array}$ & $\begin{array}{c}f_{i} \\
{[37]}\end{array}$ & $\begin{array}{c}f_{i} \\
{[34]}\end{array}$ & $\begin{array}{c}f_{i} \\
\text { [This work] }\end{array}$ \\
\hline & \multicolumn{5}{|c|}{ A-C Bond } & \multicolumn{5}{|c|}{ B-C Bond } \\
\hline $\mathrm{AgGaS}_{2}$ & 0.867 & 0.849 & 0.86 & 0.835 & 0.770 & 0.57 & 0.559 & 0.54 & 0.558 & 0.563 \\
\hline $\mathrm{AgGaSe}_{2}$ & 0.852 & 0.851 & 0.85 & 0.842 & 0.820 & 0.561 & 0.566 & 0.54 & 0.565 & 0.606 \\
\hline $\mathrm{AgGaTe}_{2}$ & & 0.853 & 0.86 & 0.851 & 0.862 & & 0.573 & 0.51 & 0.576 & 0.641 \\
\hline $\mathrm{AgInS}_{2}$ & & 0.847 & 0.85 & 0.831 & 0.770 & & 0.569 & 0.61 & 0.569 & 0.606 \\
\hline $\mathrm{AgInSe}_{2}$ & 0.846 & 0.85 & 0.85 & 0.84 & 0.820 & 0.604 & 0.573 & 0.6 & 0.575 & 0.641 \\
\hline $\mathrm{AgInTe}_{2}$ & & 0.853 & 0.84 & 0.852 & 0.862 & & 0.578 & 0.58 & 0.584 & 0.671 \\
\hline $\mathrm{ZnSiP}_{2}$ & 0.438 & 0.493 & & 0.528 & 0.509 & 0.117 & 0.2088 & & 0.197 & 0.178 \\
\hline $\mathrm{ZnGeP}_{2}$ & 0.442 & 0.493 & & 0.529 & 0.509 & 0.219 & 0.2112 & & 0.198 & 0.199 \\
\hline $\mathrm{ZnSnP}_{2}$ & 0.455 & 0.495 & & 0.53 & 0.509 & 0.298 & 0.2162 & & 0.202 & 0.214 \\
\hline $\mathrm{ZnSiAs}_{2}$ & 0.436 & 0.498 & & 0.533 & 0.554 & 0.22 & 0.2122 & & 0.199 & 0.199 \\
\hline $\mathrm{ZnGeAs}_{2}$ & 0.446 & 0.499 & & 0.534 & 0.554 & 0.182 & 0.214 & & 0.2 & 0.214 \\
\hline $\mathrm{ZnSnAs}_{2}$ & 0.45 & 0.5 & & 0.535 & 0.554 & 0.135 & 0.218 & & 0.203 & 0.227 \\
\hline $\mathrm{CdSiP}_{2}$ & 0.539 & 0.502 & & 0.538 & 0.554 & 0.191 & 0.2085 & & 0.197 & 0.178 \\
\hline $\mathrm{CdGeP}_{2}$ & 0.532 & 0.502 & & 0.538 & 0.554 & 0.231 & 0.2113 & & 0.198 & 0.199 \\
\hline $\mathrm{CdSnP}_{2}$ & 0.536 & 0.504 & & 0.539 & 0.554 & 0.298 & 0.2163 & & 0.202 & 0.214 \\
\hline $\mathrm{CdSiAs}_{2}$ & 0.553 & 0.506 & & 0.542 & 0.589 & 0.234 & 0.2202 & & 0.199 & 0.199 \\
\hline $\mathrm{CdGeAs}_{2}$ & 0.549 & 0.506 & & 0.542 & 0.589 & 0.199 & 0.2144 & & 0.2 & 0.214 \\
\hline $\mathrm{CdSnAs} 2$ & 0.553 & 0.501 & & 0.543 & 0.589 & 0.148 & 0.2184 & & 0.203 & 0.227 \\
\hline
\end{tabular}

Table 4. This table presents the values of electronic susceptibility $\chi$ for $\mathrm{A}-\mathrm{C}$ and $\mathrm{B}-\mathrm{C}$ bonds of ternary tetrahedral $\left(\mathrm{A}^{\mathrm{I}} \mathrm{B}^{\mathrm{III}} \mathrm{C}_{2}^{\mathrm{VI}}\right.$ and $\mathrm{A}^{\mathrm{II}} \mathrm{B}^{\mathrm{IV}} \mathrm{C}_{2}^{\mathrm{V}}$ ) semiconductors and plasmon energy $\hbar \omega_{\mathrm{p}}$ are taken from $[35,36]$.

\begin{tabular}{|c|c|c|c|c|c|c|c|c|c|c|}
\hline \multirow{2}{*}{ Solids } & $\hbar \omega_{p}$ & $\begin{array}{c}\chi \\
{[14]}\end{array}$ & $\begin{array}{c}\chi \\
{[35]}\end{array}$ & $\begin{array}{c}\chi \\
{[34]}\end{array}$ & $\begin{array}{c}\chi \\
\text { [This work] }\end{array}$ & $\hbar \omega_{p}$ & $\begin{array}{c}\chi \\
{[14]}\end{array}$ & $\begin{array}{c}\chi \\
{[35]}\end{array}$ & $\begin{array}{c}\chi \\
{[34]}\end{array}$ & $\begin{array}{c}\chi \\
\text { [This work] }\end{array}$ \\
\hline & \multicolumn{5}{|c|}{ A-C Bond } & \multicolumn{5}{|c|}{ B-C Bond } \\
\hline $\mathrm{CuAlS}_{2}$ & 25.110 & & 4.584 & 4.591 & 3.765 & 18.295 & & 4.955 & 5.997 & 3.942 \\
\hline $\mathrm{CuAlSe}_{2}$ & 23.231 & & 5.24 & 4.966 & 4.511 & 17.041 & & 5.908 & 6.347 & 5.181 \\
\hline $\mathrm{CuAlTe}_{2}$ & 20.859 & & 5.262 & 5.427 & 4.676 & 15.518 & & 7.102 & 6.92 & 5.678 \\
\hline $\mathrm{CuGaS}_{2}$ & 23.626 & 4.43 & 4.248 & 4.872 & 3.333 & 18.163 & 6.07 & 5.485 & 6.021 & 5.886 \\
\hline $\mathrm{CuGaSe}_{2}$ & 22.176 & 5.76 & 4.838 & 5.107 & 4.111 & 16.906 & 7.04 & 6.573 & 6.471 & 6.739 \\
\hline $\mathrm{CuGaTe}_{2}$ & 20.703 & & 5.305 & 5.434 & 4.607 & 15.195 & & 7.951 & 6.971 & 6.718 \\
\hline $\mathrm{CuInS}_{2}$ & 22.231 & 5.53 & 4.824 & 5.132 & 2.951 & 16.453 & 5.99 & 6.537 & 6.57 & 6.383 \\
\hline $\mathrm{CuInSe}_{2}$ & 23.061 & & 4.607 & 4.98 & 4.445 & 15.489 & & 7.867 & 6.984 & 6.981 \\
\hline CuInTe2 & 20.167 & & 6.939 & 5.588 & 4.371 & 14.332 & & 9.114 & 7.399 & 7.076 \\
\hline $\mathrm{AgAlS}_{2}$ & 21.536 & & 4.138 & 5.24 & 3.877 & 18.708 & & 4.829 & 5.929 & 4.122 \\
\hline $\mathrm{AgAlSe}_{2}$ & 20.090 & & 4.767 & 5.546 & 4.338 & 17.51 & & 5.461 & 6.241 & 5.470 \\
\hline $\mathrm{AgAlTe}_{2}$ & 18.681 & & 5.349 & 5.871 & 4.584 & 15.408 & & 7.073 & 6.937 & 5.598 \\
\hline $\mathrm{AgGaS}_{2}$ & 21.201 & 3.68 & 4.209 & 5.337 & 3.757 & 18.598 & 5.84 & 5.338 & 5.971 & 6.171 \\
\hline $\mathrm{AgGaSe}_{2}$ & 20.110 & 4.28 & 4.762 & 5.557 & 4.346 & 17.193 & 7.04 & 6.443 & 6.348 & 6.970 \\
\hline $\mathrm{AgGaTe}_{2}$ & 18.905 & & 5.275 & 5.831 & 4.695 & 15.149 & & 7.978 & 7.044 & 6.677 \\
\hline $\mathrm{AgInS}_{2}$ & 21.979 & & 4.048 & 5.19 & 4.038 & 16.313 & & 6.601 & 6.571 & 6.275 \\
\hline $\mathrm{AgInSe}_{2}$ & 20.513 & 4.53 & 4.658 & 5.51 & 4.522 & 15.291 & 7.41 & 7.888 & 6.946 & 6.803 \\
\hline $\mathrm{AgInTe}_{2}$ & 18.751 & & 5.325 & 5.915 & 4.619 & 13.86 & & 9.494 & 7.596 & 6.617 \\
\hline $\mathrm{ZnSiP}_{2}$ & 15.294 & & & 5.963 & 5.575 & 18.757 & & & 11.789 & 12.562 \\
\hline $\mathrm{ZnGeP}_{2}$ & 15.198 & & & 6.036 & 5.505 & 17.944 & & & 12.283 & 17.415 \\
\hline $\mathrm{ZnSnP}_{2}$ & 14.907 & & & 6.091 & 5.296 & 16.203 & & & 13.3 & 18.766 \\
\hline $\mathrm{ZnSiAs}_{2}$ & 14.473 & & & 6.242 & 6.990 & 17.62 & & & 12.414 & 16.792 \\
\hline ZnGeAs2 & 14.316 & & & 6.3 & 6.839 & 16.997 & & & 12.788 & 20.651 \\
\hline $\mathrm{ZnSnAs}_{2}$ & 14.094 & & & 6.38 & 6.628 & 15.524 & & & 13.78 & 21.257 \\
\hline
\end{tabular}




\begin{tabular}{|l|c|l|l|c|c|c|c|c|c|c|c|}
\hline $\mathrm{CdSiP}_{2}$ & 13.659 & & & 6.55 & 6.225 & & 18.845 & & & 11.74 & 12.681 \\
$\mathrm{CdGeP}_{2}$ & 13.675 & & & 6.54 & 6.240 & & 17.905 & & & 12.249 & 17.340 \\
$\mathrm{CdSnP}_{2}$ & 13.446 & & & 6.633 & 6.033 & & 16.194 & & & 13.309 & 18.746 \\
$\mathrm{CdSiAs}_{2}$ & 13.050 & & & 6.799 & 7.306 & & 17.642 & & & 12.4 & 16.834 \\
$\mathrm{CdGeAs}_{2}$ & 13.035 & & & 6.805 & 7.290 & & 16.85 & & & 12.88 & 20.295 \\
$\mathrm{CdSnAs}_{2}$ & 12.831 & & & 6.894 & 7.063 & & 15.388 & & & 13.882 & 20.886 \\
\hline
\end{tabular}

Table 5. This table presents the values of dielectric constant $\epsilon$ for $\mathrm{A}-\mathrm{C}$ and $\mathrm{B}-\mathrm{C}$ bonds of ternary tetrahedral $\left(\mathrm{A}^{\mathrm{I}} \mathrm{B}^{\mathrm{III}} \mathrm{C}_{2}^{\mathrm{VI}}\right.$ and $\left.\mathrm{A}^{\mathrm{II}} \mathrm{B}^{\mathrm{IV}} \mathrm{C}_{2}^{\mathrm{V}}\right)$ semiconductors.

\begin{tabular}{|c|c|c|c|c|c|c|c|c|}
\hline \multirow{2}{*}{ Solids } & $\begin{array}{c}\epsilon \\
{[14]}\end{array}$ & $\begin{array}{c}\epsilon \\
{[35,36]}\end{array}$ & $\begin{array}{c}\epsilon \\
{[34]}\end{array}$ & $\begin{array}{c}\epsilon \\
\text { [This work] }\end{array}$ & $\begin{array}{c}\epsilon \\
{[14]}\end{array}$ & $\begin{array}{c}\epsilon \\
{[35,36]}\end{array}$ & $\begin{array}{c}\epsilon \\
{[34]}\end{array}$ & $\begin{array}{c}\epsilon \\
{[\text { This work] }}\end{array}$ \\
\hline & \multicolumn{4}{|c|}{ A-C Bond } & \multicolumn{4}{|c|}{ B-C Bond } \\
\hline $\mathrm{CuAlS}_{2}$ & & & 5.591 & 4.765 & & & 6.997 & 4.942 \\
\hline $\mathrm{CuAlSe}_{2}$ & & & 5.966 & 5.511 & & & 7.347 & 6.181 \\
\hline $\mathrm{CuAlTe}_{2}$ & & & 6.427 & 5.676 & & & 7.92 & 6.678 \\
\hline $\mathrm{CuGaS}_{2}$ & 5.43 & & 5.872 & 4.333 & 7.07 & & 7.021 & 6.886 \\
\hline $\mathrm{CuGaSe}_{2}$ & 6.76 & & 6.107 & 5.111 & 8.04 & & 7.471 & 7.739 \\
\hline $\mathrm{CuGaTe}_{2}$ & & & 6.434 & 5.607 & & & 7.971 & 7.718 \\
\hline $\mathrm{CuInS}_{2}$ & 6.53 & & 6.132 & 3.951 & 6.99 & & 7.57 & 7.383 \\
\hline $\mathrm{CuInSe}_{2}$ & & & 5.98 & 5.445 & & & 7.894 & 7.981 \\
\hline $\mathrm{CuInTe}_{2}$ & & & 6.588 & 5.371 & & & 8.399 & 8.076 \\
\hline $\mathrm{AgAlS}_{2}$ & & & 6.24 & 4.877 & & & 6.929 & 5.122 \\
\hline $\mathrm{AgAlSe}_{2}$ & & & 6.546 & 5.338 & & & 7.241 & 6.470 \\
\hline $\mathrm{AgAlTe}_{2}$ & & & 6.871 & 5.584 & & & 7.937 & 6.598 \\
\hline $\mathrm{AgGaS}_{2}$ & 4.68 & & 6.337 & 4.757 & 6.84 & & 6.971 & 7.171 \\
\hline $\mathrm{AgGaSe}_{2}$ & 5.28 & & 6.557 & 5.346 & 8.04 & & 7.348 & 7.970 \\
\hline $\mathrm{AgGaTe}_{2}$ & & & 6.831 & 5.695 & & & 8.044 & 7.677 \\
\hline $\mathrm{AgInS}_{2}$ & & & 6.19 & 5.038 & & & 7.571 & 7.275 \\
\hline $\mathrm{AgInSe}_{2}$ & 5.53 & & 6.51 & 5.522 & 8.41 & & 7.946 & 7.803 \\
\hline $\mathrm{AgInTe}_{2}$ & & & 6.915 & 5.619 & & & 8.596 & 7.617 \\
\hline $\mathrm{ZnSiP}_{2}$ & 6.84 & 6.486 & 6.963 & 6.575 & 10.41 & 10.931 & 12.789 & 13.562 \\
\hline $\mathrm{ZnGeP}_{2}$ & 6.84 & 6.526 & 7.036 & 6.505 & 11.55 & 11.494 & 13.283 & 18.415 \\
\hline $\mathrm{ZnSnP}_{2}$ & 6.84 & 6.648 & 7.091 & 6.296 & 12.65 & 12.93 & 14.3 & 19.766 \\
\hline $\mathrm{ZnSiAs}_{2}$ & 7.95 & 6.839 & 7.242 & 7.990 & 11.8 & 11.731 & 13.414 & 17.792 \\
\hline $\mathrm{ZnGeAs}_{2}$ & 7.95 & 6.913 & 7.3 & 7.839 & 14.29 & 12.223 & 13.788 & 21.651 \\
\hline $\mathrm{ZnSnAs}_{2}$ & 7.95 & 7.021 & 7.38 & 7.628 & 18.08 & 13.568 & 14.78 & 22.257 \\
\hline $\mathrm{CdSiP}_{2}$ & 6.99 & 7.239 & 7.55 & 7.225 & 10.41 & 10.874 & 12.74 & 13.681 \\
\hline $\mathrm{CdGeP}_{2}$ & 6.99 & 7.232 & 7.54 & 7.240 & 11.55 & 11.521 & 13.249 & 18.340 \\
\hline $\mathrm{CdSnP}_{2}$ & 6.99 & 7.355 & 7.633 & 7.033 & 12.65 & 12.922 & 14.309 & 19.746 \\
\hline CdSiAs2 & 8.22 & 7.579 & 7.799 & 8.306 & 11.8 & 11.603 & 13.4 & 17.834 \\
\hline $\mathrm{CdGeAs}_{2}$ & 8.22 & 7.588 & 7.805 & 8.290 & 14.29 & 12.343 & 13.88 & 21.295 \\
\hline $\mathrm{CdSnAs}_{2}$ & 8.22 & 7.73 & 7.894 & 8.063 & 18.08 & 13.71 & 14.882 & 21.886 \\
\hline
\end{tabular}

SUMMARY AND CONCLUSION

It is obvious from the results introduced in the tables 1 to 5 that the values obtained for the proposed properties for ternary tetrahedral semiconductors are in close agreement to the values reported so far for the same properties by earlier researchers. So, we draw an inference that average atomic number in addition to product of ionic charges of the elements in a compound plays a vital role in determining the electronic and optical properties of the ternary tetrahedral $\left(\mathrm{A}^{\mathrm{I}} \mathrm{B}^{\mathrm{III}} \mathrm{C}_{2}^{\mathrm{VI}}\right.$ and $\mathrm{A}^{\mathrm{II}} \mathrm{B}^{\mathrm{IV}} \mathrm{C}_{2}^{\mathrm{V}}$ ) compounds. The proposed empirical relations for the ionic gap ( $\mathrm{E}_{\mathrm{c}}$ ) and average energy gap $\left(\mathrm{E}_{\mathrm{g}}\right)$ have been found inversely related to average atomic number and the product of ionic charges of the elements in the ternary tetrahedral semiconductors. The crystal ionicity $\left(f_{i}\right)$ is directly related to the average atomic number and inversely to the product of ionic charges of the elements in the compound, while the electronic susceptibility $(\chi)$ and dielectric constant $(\epsilon)$ are directly related to both the criterion. It is noteworthy that the proposed relations are simple and widely applicable and the values obtained for electronic and optical properties are in accordance to the earlier 
workers reported. The new idea conceptualized herein will be encouraging to the material scientists for exploring new homologous compounds with desired ionic gap $\left(\mathrm{E}_{\mathrm{c}}\right)$, average energy gap $\left(\mathrm{E}_{\mathrm{g}}\right)$, crystal ionicity ( $\left.\mathrm{f}_{\mathrm{i}}\right)$, electronic susceptibility $(\chi)$ and dielectric constant $(\epsilon)$, which may revolutionized modern microelectronic industry.

\section{ORCID IDs}

Dajesh C. Gupta, https://orcid.org/0000-0002-0560-7077; (D Khushvant Singh, https://orcid.org/0000-0002-1957-4384

(D) Ajay S. Verma, https://orcid.org/0000-0001-8223-7658

\section{REFERENCES}

[1] Neeraj, Pravesh, R. Gautam, S. Pal, C. Mohan, S. Kumari, S.R. Bhardwaj, and A.S. Verma, J. Nanoelectronics and Optoelectronics, 14, 759 (2019), https://doi.org/10.1166/jno.2019.2553.

[2] S. Tomar, R. Gautam, Pravesh, C. Mohan, S.K. Gupta, S.R. Bhardwaj, and A.S. Verma, Chalcognide Letters, 16, 1 (2019), https://chalcogen.ro/1_TomarS.pdf.

[3] J.L. Shay, and J.H. Wernick, Ternary Chalcopyrite Semi-conductors: Growth, Electronic Properties and Applications (Pergamon Press, Oxford, 1975), pp.11, 12 and 73.

[4] N. Yamamoto, Ph.D. Thesis, University of Osaka, Japan (1976).

[5] J.E. Jaffe, and A. Zunger, Phys. Rev. B, 20, 1882 (1984), https://doi.org/10.1103/PhysRevB.29.1882.

[6] R. Marquez, and C. Rincon, Phys. Stat. Sol. (b), 191, 115 (1995), https://doi.org/10.1002/pssb.2221910112.

[7] M.I. Alonso, K. Wakita, J. Pascual, and N. Yamamoto, Phys. Rev. B, 63, $75203 \quad$ (2001), https://doi.org/10.1103/PhysRevB.63.075203.

[8] Xiaoshu Jiang, and W.R.L. Lambrecht, Phys. Rev. B, 69, 035201 (2004), https://doi.org/10.1103/PhysRevB.69.035201.

[9] F. Chiker, B. Abbar, A. Tadjer, S. Bresson, B. Khelifa, and C. Mathieu, Physica B, 349, 181 (2004), https://doi.org/10.1016/j.physb.2004.03.087.

[10] V. Kumar, and B.S.R. Sastry, J. Phys. Chem. Solids, 66, 99 (2005), https://doi.org/10.1016/j.jpcs.2004.08.034.

[11] A.H. Reshak, Physica B, 369, 243 (2005), https://doi.org/10.1016/j.physb.2005.08.038.

[12] L.K. Samanta, and S. Chaterjee, Infrared Phys. Technol. 46, 370 (2005), https://doi.org/10.1016/j.infrared.2004.06.009.

[13] A. Chahed, O. Benhelal, H. Rozale, S. Laksari, and N. Abbouni, Phys. Status Solidi B, 244, 629 (2007), https://doi.org/10.1002/pssb.200642050.

[14] B.F. Levine, Phys. Rev. B, 7, 2591, 2600 (1973), https://doi.org/10.1103/PhysRevB.7.2591.

[15] J.A. van Vechten, Phys. Rev. 182, 891 (1969), https://doi.org/10.1103/PhysRev.182.891.

[16] J.C. Phillips, and J.A. Van Vechten, Phys. Rev. B, 2, 2147 (1970), https://doi.org/10.1103/PhysRevB.2.2147.

[17] J.C. Phillips, Rev. Mod. Phys. 42, 317 (1970), https://doi.org/10.1103/RevModPhys.42.317.

[18] D.R. Penn, Phys. Rev. 128, 2093 (1962), https://doi.org/10.1103/PhysRev.128.2093.

[19] O.P. Singh, and V.P. Gupta, Phys. Stat. Sol. (b), 137, 97 (1986), https://doi.org/10.1002/pssb.2221370112.

[20] V. Kumar, and G.M. Prasad, J. Phys. Chem. Solids, 50, 899 (1989), https://doi.org/10.1016/0022-3697(89)90037-1.

[21] V. Kumar Srivastava, J. Phys. C: Solid State Phys. 19, 5689 (1986), https://doi.org/10.1088/0022-3719/19/28/019.

[22] V. Kumar Srivastsva, Phys. Rev. B, 29, 6993 (1984), https://doi.org/10.1103/PhysRevB.29.6993.

[23] A.S. Verma, Solid State Commun. 149, 1236 (2009), https://doi.org/10.1016/j.ssc.2009.04.011.

[24] L. Pauling, The Nature of Chemical Bond (Ithaca, NY: Cornell University Press, 1906).

[25] L. Marton, L.B. Leder, and H. Mendlowitz, in: Advances in Electronics and Electron Physics, Vol. 7, edited by L. Marton (Academic Press, New York, 1955), p. 225.

[26] H.R. Philipp, and H. Ehrenreich, Phys. Rev. 129, 1530 (1963), https://doi.org/10.1103/PhysRev.129.1550.

[27] H. Raether, Ergeb, Exakten Naturwiss. (Germany) 38, 84 (1965).

[28] C. Kittel, Introduction to Solid State Physics, $4^{\text {th }}$ ed. (Wiley, New York, 1971), (Second Wiley Eastern Re-print, New Delhi, 1974), pp. 227.

[29] J.C. Phillips, Bonds and Bands in Semiconductors (Academic Press, New York, (1973).

[30] O.P. Singh, and V.P. Gupta, Phys. Status Solidi (b), 129, K153, (1985).

[31] A. Jayaraman, B. Batlogg, R.G. Maines, and H. Bach, Phys. Rev. B, 26, 3347 (1982), https://doi.org/10.1103/PhysRevB.26.3347.

[32] D.B. Srideshmukh, and K.G. Subhadra, J. Appl. Phys. 59, 276 (1986), https://doi.org/10.1063/1.336826.

[33] K.S. Krishnan, and S.K. Roy, Proc. R. Soc. London, 210, 481 (1952), https://doi.org/10.1098/rspa.1952.0014.

[34] A.S. Verma, and S.R. Bhardwaj, Phys. Stat. Sol. (b), 243, 4025 (2006), https://doi.org/10.1002/pssb.200642229.

[35] V. Kumar, J. Phys. Chem. Solids, 48, 827 (1987), https://doi.org/10.1016/0022-3697(87)90033-3.

[36] V. Kumar Srivastava, Phys. Rev. B, 36, 5044 (1987), https://doi.org/10.1103/PhysRevB.36.5044.

[37] H. Neumann, Cryst. Res. Technol. 18, 1299, 1391, 665, 901 (1983), https://doi.org/10.1002/crat.2170181016.

\section{ОПТОЕЛЕКТРОННІ ВЛАСТИВОСТІ ПОТРІЙНИХ ТЕТРАЕДРИЧНИХ НАПІВПРОВІДНИКІВ}

P.C. Гупта ${ }^{a}$, А.С. Верма ${ }^{\text {, }}$ К. Сінгх ${ }^{\mathrm{a}}$

${ }^{a}$ Фізичний факультет, коледж Б.С.А., Матхура, 281004 (Індія)

${ }^{b}$ Факультет природничих та прикладних наук, Школа технологій, Університет Глокал, Сахаранпур 247121 (Індія)

Діелектрична інтерпретація іонності кристалів, розроблена Філіпсом та Ван Вехтеном (P.V.V), була використана для оцінки різних властивостей основного стану для широкого спектра напівпровідників та ізоляторів. Однак актуальність теорії діелектриків P.V.V. обмежена лише простими структурованими сполуками $\mathrm{A}^{\mathrm{N}} \mathrm{B}^{8-\mathrm{N}}$, які мають певний зв'язок. Левін розширив P.V.V. теорію іонності для кристалів з багатьма зв'язками та комплексних кристалів та оцінив багато параметрів зв'язку для потрійних тетраедричних напівпровідників. Деякі інші дослідники розширили роботу Левіна за допомогою концепції іонного заряду та відстані до найближчого оточення на бінарні та потрійні тетраедричні кристали для оцінки властивостей основного стану. У цій роботі для розуміння деяких електронних та оптичних властивостей, таких як іонний зазор $\left(\mathrm{E}_{\mathrm{c}}\right)$, середня енергетична щілина $\left(\mathrm{E}_{\mathrm{g}}\right)$, іонність кристалів $\left(\mathrm{f}_{\mathrm{i}}\right)$, електронна сприйнятливість $(\chi)$ та діелектрична проникність $(\epsilon)$ потрійних чотиригранних напівпровідників $\left(\mathrm{A}^{\mathrm{II}} \mathrm{B}^{\mathrm{IV}} \mathrm{C}_{2}^{\mathrm{V}}\right.$ and $\left.\mathrm{A}^{\mathrm{I}} \mathrm{B}^{\mathrm{III}} \mathrm{C}_{2}^{\mathrm{VI}}\right)$ була використана нова гіпотеза середнього атомного числа елементів у сполуках. Відмічається досить прийнятна відповідність оцінених значень 3 оцінками зробленими інших іншими дослідниками.

КЛЮЧОВІ СЛОВА: іонність кристалів, середній атомний номер, халькопірити 